\title{
A matched solution method for the prediction of residual stresses for flat rolling
}

\author{
A. Dixon* $\quad$ W. Y. D. Yuen ${ }^{\dagger}$
}

(Received 8 August 2003, revised 30 January 2004)

\begin{abstract}
Steel strip must meet tight dimensional tolerances as well as having good 'flatness' such that the strip is planar when not subject to external forces. This latter attribute demands extremely small residual stresses. A computational model is developed to describe the lateral variations of the magnitude and direction of the plastic deformation of the strip during rolling, together with the corresponding strip stresses and the degree of elastic roll flattening. In addition the downstream lateral variation in longitudinal strains, and resultant stresses, are calculated. From these the strip flatness can be predicted. It was found that for thin, wide strip the procedure became numerically unstable. This was overcome by developing an analytical solution for the simpler rolling conditions, away from the strip edge, and iteratively matching
\end{abstract}

*BlueScope Steel Research, P.O. Box 202, Port Kembla, NSW, 2505, Australia. mailto: Andrew. Dixon@bluescopesteel.com

$\dagger$ BlueScope Steel Research. mailto:Daniel. Yuen@bluescopesteel.com

See http://anziamj.austms.org.au/V45/CTAC2003/Dixo for this article, C Austral. Mathematical Soc. 2004. Published June 11, 2004. ISSN 1446-8735 
this solution to the numerical solution found for the edge region. This provided a more stable solution procedure.

\section{Contents}

1 Introduction

C436

2 Mathematical Description and Solution Procedure

C438

3 Matched Domain Procedure

C445

References

C447

\section{Introduction}

Steel strip is produced by the repeated rolling of hot steel slabs typically $200 \mathrm{~mm}$ thick, to the required thickness. The final product must meet tight dimensional tolerances in width and thickness as well as in the flatness (strip shape). The latter is reflected in the residual strains in the strip after rolling, with typical tolerances for the residual strain to be less than $0.01 \%$. During rolling there is generally some lateral spread, causing a width change, together with variation in strip thickness reduction across the width, due to the bending and flattening of the mill rolls. Both these effects generate residual strains. An understanding of these mechanisms under different rolling conditions is desirable, together with the ability to predict the residual strains and spread for the lateral variation in thickness reduction.

For the flat rolling of thin strip the residual and lateral strains are small compared to the thickness reduction, making the study of these strains difficult using numerical techniques, such as the finite element method, due to 


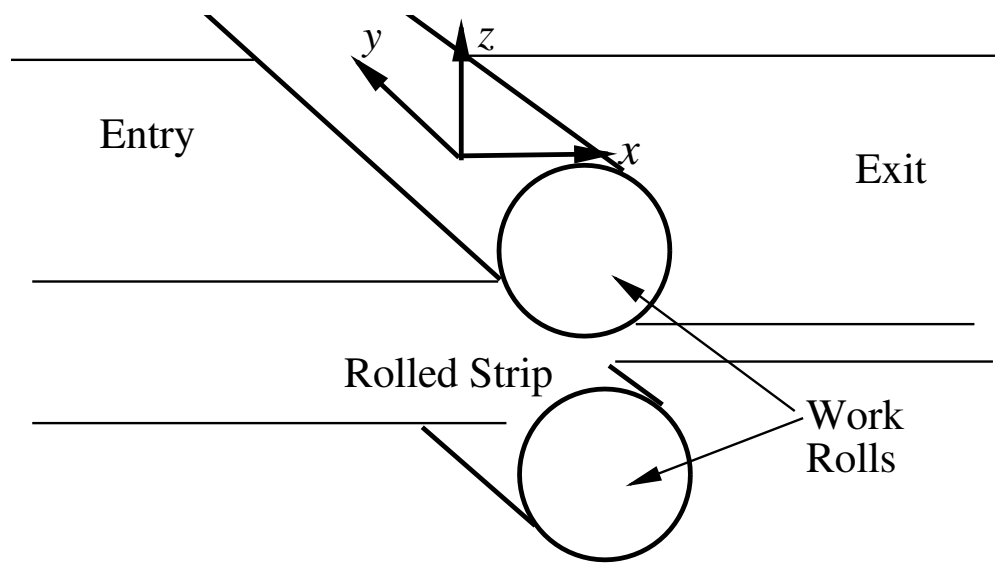

FiguRE 1: Strip thickness reduction for rolling (not to scale).

limited arithmetic precision. These numerical methods have either concentrated on plane strain cases, valid in the central regions of thin strip, or the lateral spread of thick strip. The analysis of lateral variation of strains for thin strip rolling has been studied mainly using analytical-numerical techniques. Although the residual strains in the strip after rolling are small, if the strip is kept flat downstream under tension large residual stresses can be created due to the large elastic modulus of steel, the latter being typically two to three orders of magnitude larger than the yield stress. The effect of these downstream stresses on the rolling region stress field should be included in a physically realistic model for thin strip rolling, as has been done by Tozawa [1]. Stress and strain solutions were then found for strip of moderate thicknesses using numerical methods, after analytical approximations to the plastic flow and stress fields. Very little has been published for the case of thin strip. In the present study it was found that when the strip is thin, of thicknesses typical for the last stands of a hot strip mill, these methods become unstable as the inner boundary condition at the strip centre is difficult to satisfy due to the solution becoming divergent. This could be overcome by 
the use of an inner solution, found under appropriate approximations for this region, and which exactly satisfies the inner boundary condition. A solution valid across the whole strip is then obtained by ensuring solution continuity across the boundary between the inner and outer regions.

\section{Mathematical Description and Solution Procedure}

For a given stress field plastic strain rates are given by the Levy-Mises equations

$$
\frac{D \epsilon_{x} / D t}{\sigma_{x}-\bar{\sigma}}=\frac{D \epsilon_{y} / D t}{\sigma_{y}-\bar{\sigma}}=\frac{D \epsilon_{z} / D t}{\sigma_{z}-\bar{\sigma}}
$$

where the directions of $x, y$ and $z$ are shown in Figure $1, \epsilon_{x}, \epsilon_{y}, \epsilon_{z}$ and $\sigma_{x}$, $\sigma_{y}, \sigma_{z}$ are the corresponding strains and normal stresses respectively in the three axial directions, $\bar{\sigma}=\frac{1}{3}\left(\sigma_{x}+\sigma_{y}+\sigma_{z}\right)$ is minus the hydrostatic pressure.

The term $D$ is the material time derivative. For plastic deformation to occur the stresses within the strip must satisfy a yielding condition such as that of von Mises

$$
\left(\sigma_{x}-\sigma_{y}\right)^{2}+\left(\sigma_{x}-\sigma_{z}\right)^{2}+\left(\sigma_{z}-\sigma_{y}\right)^{2} \approx \frac{3}{2} Y^{2},
$$

where $Y$ is the yield stress. The effects of the shear stresses have been neglected, as will be discussed later. To simplify the analysis it is common to assume [1] the relation

$$
\frac{D \epsilon_{y}}{D t}=\alpha(y) \frac{D \epsilon_{y}}{D t}
$$

As plastic deformation is incompressible one obtains the relation

$$
(1+\alpha) \epsilon_{x}^{p}+\epsilon_{z}^{p}=0 \quad \text { with } \quad \epsilon_{y}^{p}=\alpha \epsilon_{x}^{p},
$$


after integrating through the plastic region between the rolls in the rolling direction, where $\epsilon_{y}^{p}$ and $\epsilon_{x}^{p}$ are the plastic strains in the corresponding directions, generated from rolling. For thin strip one can write $\epsilon_{z}^{p}=\ln (H / h)=$ $-\ln (1-r)$ where $H$ and $h$ are the strip entry and exit thicknesses and $r=(H-h) / H$ is the thickness reduction.

The parameter $\alpha$ indicates the direction of the flow of strip material between the rolls, being zero if there is no lateral flow. From Equation (4) we obtain

$$
\alpha=\left(\ln (1-r)-\epsilon_{x}^{p}\right) / \epsilon_{x}^{p} .
$$

By substituting Equation (3) into Equation (1) and by the definition of the hydrostatic pressure an expression for $\sigma_{y}$ may be found in terms of the other normal stresses. With the use of Equation (2) an expression for $\sigma_{z}$ can then be found in terms of $\sigma_{y}$ and $\sigma_{x}$ to finally give

$$
\sigma_{x}=\sigma_{y}+\frac{(1-\alpha) Y}{2 \sqrt{1+\alpha+\alpha^{2}}}
$$

where, for convenience, the effects of lateral shear stresses have been neglected. This is justifiable for strip sufficiently thin, which is the main concern of the present paper, as the resistance to lateral flow is then dominated by the surface frictional forces rather than lateral shearing force, the latter decreasing with thickness.

The lateral variation of $\sigma_{y}$ can be found from the Lateral Equilibrium Equation

$$
\frac{\partial h \sigma_{y}}{\partial y}-\sigma_{z} \frac{\partial h}{\partial y}-\frac{2 q\left(u-u_{R}\right)}{\sqrt{\left(u-u_{R}\right)^{2}+v^{2}}}=0
$$

where $u$ and $v$ are the local strip speed in the rolling direction and lateral direction respectively and $u_{R}$ is the rolling speed. The surface friction $q$ may be expressed in terms of the yield stress by $q=m Y$ where $m$ is the friction factor. The last term on the left hand side represents the lateral component of the surface friction stress. The lateral strip speed may be found from the 
lateral strain. In particular,

$$
\frac{\partial v}{\partial y}=\frac{D \epsilon_{y}}{D t} \approx u \frac{\partial \epsilon_{y}}{\partial x} \approx u \alpha \frac{\partial \epsilon_{x}}{\partial x} \quad \text { or } \quad v \approx \int_{0}^{y} u \alpha\left(\frac{\partial}{\partial x} \epsilon_{x}\right) d y^{\prime},
$$

where $y^{\prime}$ is the integration variable representing the $y$-direction. The variation in the strip speed $u$ between the rolls may be found from mass flow conservation, using Equation (4).

If, after rolling, the plastic elongation (in the rolling direction) of the downstream strip is not uniform across the strip width then elastic stresses will result if the strip is to remain flat. For such flat strip the elastic strains must vary across the strip width so that the total elastic and plastic elongation is constant. The strip can be modelled as a series of parallel ribbons in the rolling direction ( $y z$ plane), which are under plane stress in the vertical and lateral directions (that is, $x y$ and $x z$ planes). In the rolling direction the stress variation across the strip width is defined as

$$
\sigma_{x}(y)=\bar{\sigma}_{x}-E\left(\epsilon_{x}^{p}(y)-\bar{\epsilon}_{x}^{p}\right),
$$

with $\bar{\sigma}_{x}$ and $\bar{\epsilon}^{p}$ being the corresponding values at a reference point, for the present analysis this will be the strip centreline with values set. It will be assumed that this expression for downstream conditions is also valid at the rolling region exit.

The final strip thickness depends on the vertical distance between the rolls at the rolling region exit. This in turn is dependent on the degree of roll deformation per roll from flattening $\delta$, which is dependent on the lateral variation in roll pressure $p=-\sigma_{z}$. The roll flattening can be calculated from,

$$
\delta(x, y)=\frac{1-\nu^{2}}{\pi E} \iint_{A} p\left(x^{\prime}, y^{\prime}\right) \times F\left(x^{\prime}, x, y^{\prime}, y\right) d x^{\prime} d y^{\prime},
$$

where $F$ is an influence function [1] and $A$ is the area of contact between the strip and roll. The variation in strip exit thickness in the rolling region is then given by,

$$
h(x, y)=h^{0}(x)+2 \delta(x, y)
$$


where $h_{0}$ is the hypothetical thickness profile through the rolling region if there was no roll deformation.

The solutions for the final strip thickness variation across the width, together with the variation in strip stresses and strains, are found using a two level iteration process. The first, inner iteration, calculates the lateral variation in strip stresses and strains for an assumed degree of roll deformation such that the stress boundary conditions are met. The outer iteration procedure ensures the roll deformation is compatible with the pressure profile from the strip onto the rolls across the strip width.

The steps for the inner iteration is termed Procedure A and is summarised as follows:

1. The strip edge conditions, $\sigma_{y}=0$ at $y=W / 2$, is set where $W$ is the strip width. Estimates for the other stresses and $\alpha$ at the edge are found under these plane stress conditions. A value for the strip lateral spread at the edge $s_{\text {edge }}$ is set. This is an initial guess for the first iteration.

2. At a position $\Delta y$ (further) inwards the lateral stress $\sigma_{y}$ is updated from the Lateral Equilibrium Equation (7), using Euler's scheme together with the thickness profile from the assumed roll deformation.

3. The normal stress in the rolling direction $\sigma_{x}$ is calculated from Equation (6) and the normal stress $\sigma_{z}$ from Equation (2).

4. The corresponding strain in the rolling direction is found from Equation (9).

5. The corresponding value for $\alpha$ is found from Equation (5) and the lateral spread $s$ is updated using $s(y)=s_{\text {edge }}-2 \bar{\epsilon} \int_{y}^{W / 2} \alpha d y^{\prime}$.

6. Steps $2-5$ are repeated until the strip centreline is reached. 


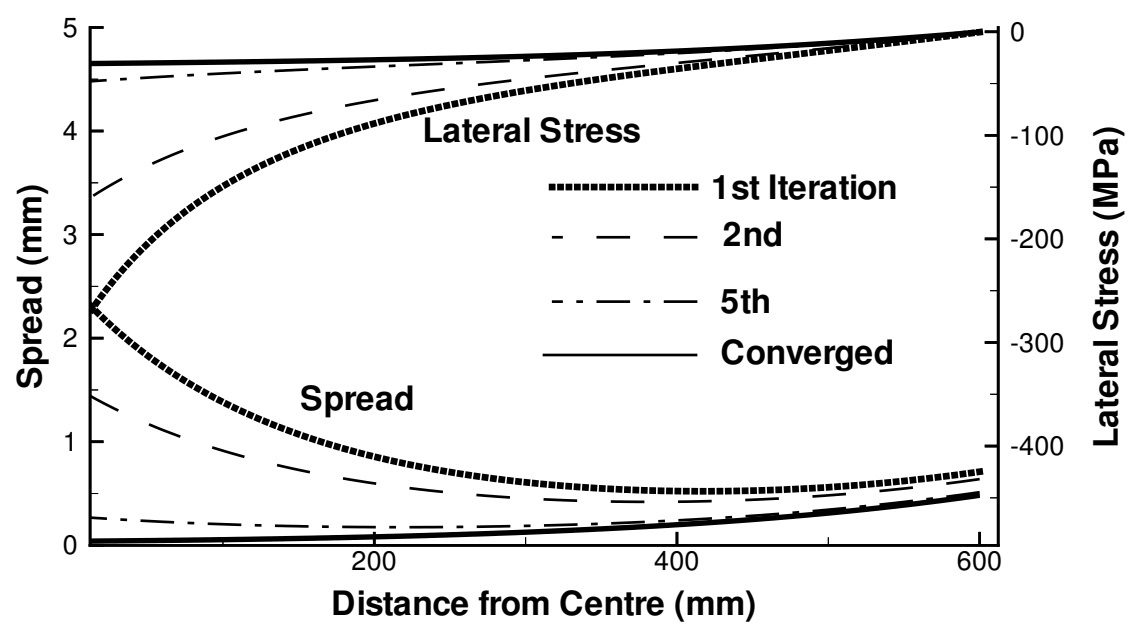

Figure 2: Calculated solutions for lateral normal stress and strip spread for 1st, 2nd, 5th iteration of Procedure A together with converges solution. Strip entry thickness is $20 \mathrm{~mm}$, thickness reduction $40 \%, Y=300 \mathrm{MPa}$ and $m=0.4$.

7. The inner boundary condition of no lateral strip movement, $s=0$ at the strip centreline $y=0$ is tested and the error value is used to update the edge lateral spread $s_{\text {edge }}$, (see Step 1), using the secant method.

8. Steps 1-7 are repeated until the inner boundary condition is satisfied.

As the rolling region is short compared to the strip width then an average value for these stresses and the strip velocity through the rolling region may be used. Typical results from the iteration process of Procedure A is shown in Figure 2 where the profile across the strip width of the lateral normal stress is shown assuming no roll deformation. Also shown is the predicted variation in lateral spread. The solutions converge to the correct inner boundary condition where there is no lateral strip spread at the strip centreline. 


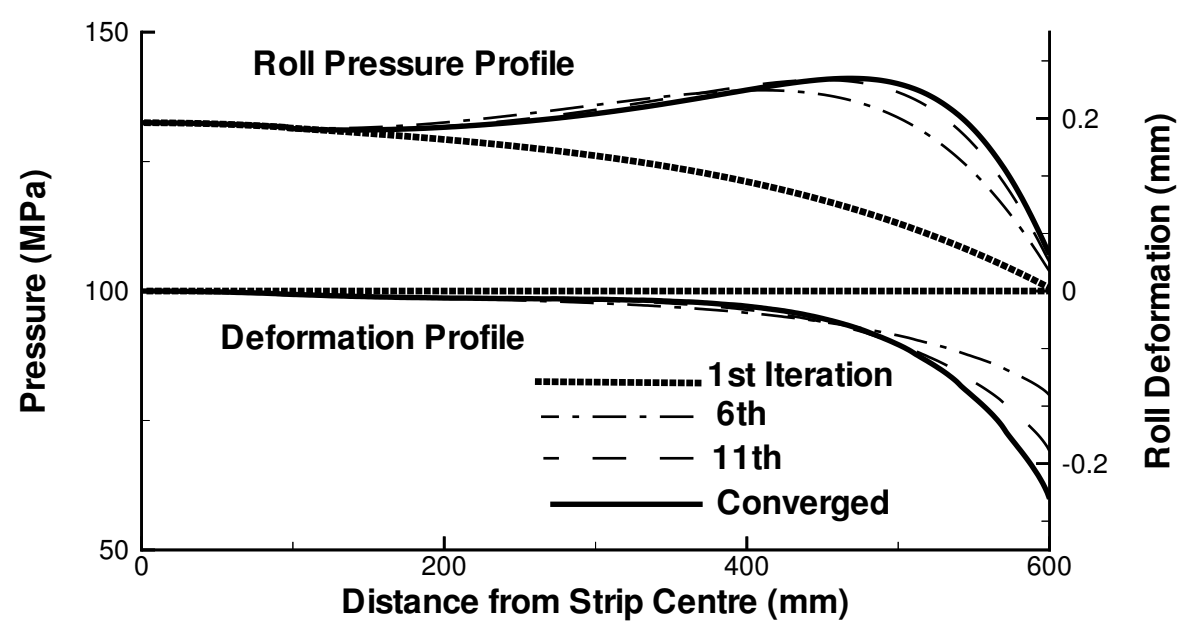

Figure 3: Calculated iterations and converged solution for roll pressure and variation in roll deformation (compared to the width centreline). Rolling conditions as in Figure 2.

Once the stresses across the strip width are known then the roll deformation can be calculated. This provides the outer iterations loop:

1. Obtain the profile of strip pressure $p=-\sigma_{z}$ from Procedure A using the strip thickness profile from the previous estimate of the roll deformation.

2. Use this pressure profile to update the roll deformation from Equation (10) and the strip thickness profile through the rolling region from Equation (11).

3. Repeat Steps 1-2 until both the stress profiles and roll deformation profiles have converged. 


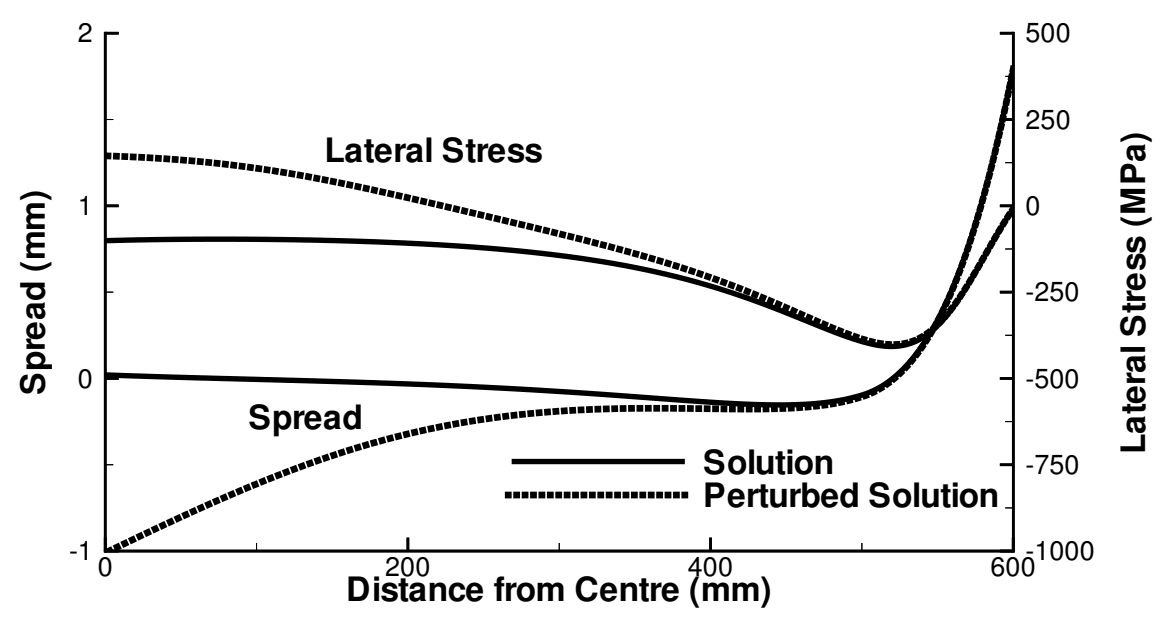

FIgURE 4: Strip lateral stress and lateral spread solutions with an entry thickness of $4 \mathrm{~mm}$ and thickness reduction of $40 \%$. Lateral speed at edge has been perturbed by a small amount to obtain large changes to the solutions in the central region.

The result of this iteration procedure is shown in Figure 3. The converged solution now shows the strip edges to be thinner than at the centreline due to the variation in roll deformation in this region. The strip pressure onto the roll initially increases away from the strip centre to a distance about $100 \mathrm{~mm}$ from the edge and then decreases with a discontinuity at the strip edge which causes the large variation in roll deformation in this region. 


\section{Matched Domain Procedure}

It was found that when the strip thickness used was decreased sufficiently the iteration Procedure A became unstable with the stress and strain solution in the strip central region becoming exponentially divergent. Impractically accurate estimates for the strip lateral spread at the edge were necessary to overcome this difficulty. This is shown in Figure 4 where a small perturbation to the strip lateral spread at the edge produced a large change to the calculated spread at the strip centre. The strip thickness entering the mill is $4 \mathrm{~mm}$ for this case, rather than $20 \mathrm{~mm}$ for the cases of Figures 2-3.

A possible method to improve the stability of the computational scheme would be to incorporate the inner boundary condition into an implicit scheme. It was felt, however, that this would be difficult due to the algorithm complexity and the non-linearity of the problem. Instead a method was devised that exploited the simpler conditions of the strip central region where there is less lateral strain, that is, $\alpha$ is small, and the variation in roll deformation can be largely ignored. An analytical solution for this region could then be obtained which was matched at some domain boundary to the numerical solution obtained using the above procedure.

Under the approximation of small $\alpha$,and using Equations (6), (9) and (5) then

$$
\frac{\partial \sigma_{y}}{\partial y} \approx \frac{\partial \sigma_{x}}{\partial y} \approx-E \frac{\partial \epsilon^{p}}{\partial y} \approx E\left(\bar{\epsilon} \frac{\partial \alpha}{\partial y}+\frac{1}{1-\bar{r}} \frac{\partial r}{\partial y}\right),
$$

which may be substituted into the Lateral Equilibrium Equation (7) to give [2]

$$
\frac{\partial^{2} \alpha}{\partial y^{2}}-G^{2} \alpha=\frac{1}{\epsilon_{x}^{0}} \frac{\partial^{2} r}{\partial y^{2}} \quad \text { with } \quad G^{2}=\frac{m Y(\partial u / \partial x)}{h E \epsilon_{x}^{0}\left|u-u_{R}\right|},
$$

where $G$ may be evaluated from the known roll profile and, again, an average value taken through the rolling region. In the present context for the inner region the thickness reduction variation across the strip is ignored and the 


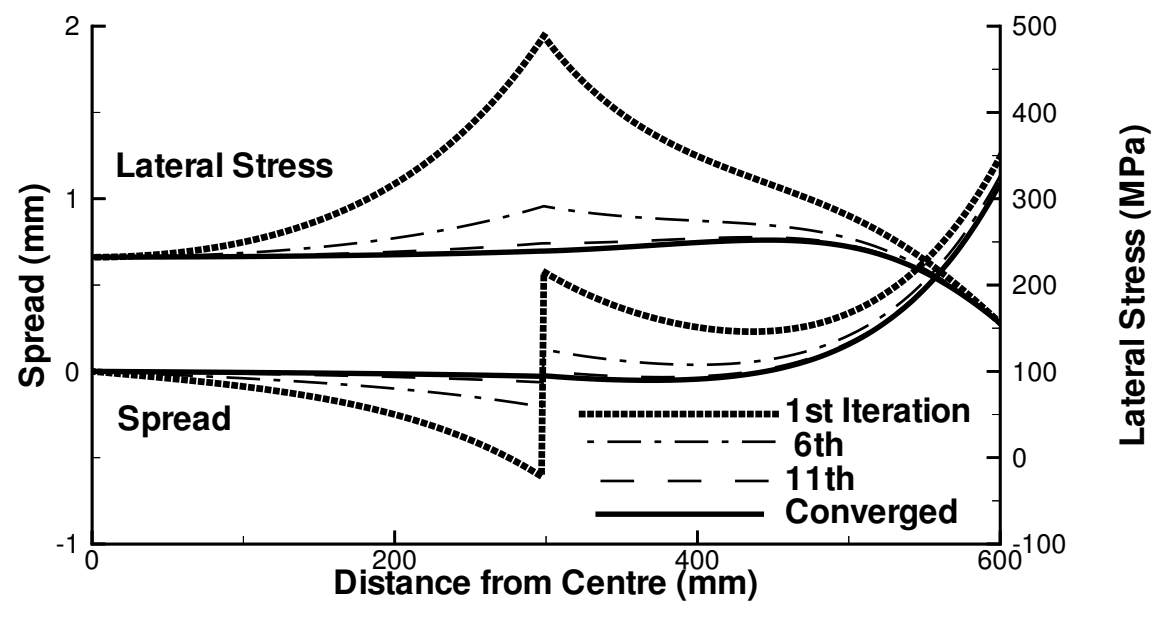

Figure 5: Pressure and lateral spread solutions for inner and outer regions. Converged solution has continuous lateral spread over boundary.

solution for $\alpha$ can be expressed as,

$$
\alpha=A \exp (G(W / 2-y))+B \exp (-G(W / 2-y)) .
$$

This solution form was chosen to explain the cause of the instability found previously for small strip thicknesses. If the factors $A$ and $B$ are chosen from conditions at the edge then any change from the correct value of $A$ will cause an error in the solution to grow exponentially towards the strip centreline. The magnitude of this solution error will increase for decreasing strip thickness, as can be seen from the definition of $G$, which is consistent with the results of the previous section. A more suitable solution form which guarantees symmetry about the strip centre and zero lateral strip velocity at the centreline is

$$
\alpha=\alpha_{0} \cosh (G y), \quad \text { giving } \quad s(y)=\frac{2 \alpha \bar{\epsilon}}{G} \sinh (G y) .
$$


This solution for the inner region is then matched to the numerical solution for the outer strip edge region at the boundary of the two regions, $y=\hat{y}$, say. If the Lateral Equilibrium Equation is numerically integrated from the strip edge inwards using the procedure of the previous section, to obtain the value of the lateral stress at the boundary to be $\sigma_{y}^{N}(\hat{y})$, (where the superscript denotes the numerical solution), then the inner analytical stress solution can be matched using Equations (6) and (9) to obtain,

$$
\alpha_{0}=\left(\frac{1}{2} Y-\bar{\sigma}_{x}-\sigma_{y}^{N}(\hat{y})\right) / E \bar{\epsilon}(1-\cosh (G \hat{y})) .
$$

Using the secant method the lateral strip spread at the edge is chosen so that the lateral strip spread is continuous across the boundary. It can be shown that this condition guarantees that the stress gradient is also continuous, as can be seen in Figure 5. To test the reliability of the method the position of the boundary between the inner and outer regions was shifted. The resulting variation in the solution was found to be small, giving confidence to the solution method.

Acknowledgments: the authors thank the Management of the BlueScope Steel Research for permission to publish the material contained in this paper.

\section{References}

[1] Tozawa, Y., Ishikawa, T. and Iwata, N. Predicting the profile of rolled strip, Steel Rolling Conference, Swansea, 1982, pp.787-796 C437, C438, C440

[2] Dixon, A.and Yuen, W. Y. D. An analytical model for the lateral spread and shape defects from the rolling of a ridge profile or edge drop, 44th MWSP Conf. Proc., Vol. XL, ISS, September 8-11, Orlando, USA, 2002, pp.267-278 C445 